\title{
Thermal analysis of montmorillonite modified by imidazolium
}

\section{Jingjie Zhang MD}

Researcher, Key Laboratory of Advanced Technology for Special Functional Materials of Ministry of Education, School of Materials Science and Engineering, Wuhan University of Technology, Wuhan, China

Qilin Mei PhD*

Professor, Key Laboratory of Advanced Technology for Special Functional Materials of Ministry of Education, School of Materials Science and Engineering, Wuhan University of Technology, Wuhan, China

\section{Long Chen MD}

Researcher, Key Laboratory of Advanced Technology for Special Functional Materials of Ministry of Education, School of Materials Science and

Engineering, Wuhan University of Technology, Wuhan, China

\section{Xia Chen MD}

Researcher, Key Laboratory of Advanced Technology for Special Functional Materials of Ministry of Education, School of Materials Science and Engineering, Wuhan University of Technology, Wuhan, China

Lei Zu PhD

Professor, Key Laboratory of Advanced Technology for Special Functional Materials of Ministry of Education, School of Materials Science and Engineering, Wuhan University of Technology, Wuhan, China

In order to improve the performance of sodium (Na) montmorillonite (MMT), in this study, 1-hexadecyl-2,3dimethylimidazolium bromide was synthesized and used as a cationic surfactant for the organic modification of sodium MMT. The modification effect of $\mathrm{pH}$ was investigated, and the structure of the imidazole organic modifier was characterized by nuclear magnetic resonance and Fourier transform infrared spectroscopy (FTIR). In addition, the modified sodium MMT was systematically confirmed by FTIR and X-ray diffraction. The results showed that the imidazolium surfactant successfully intercalated into the galleries of MMT and enlarged the (001) $d$ spacing of MMT. It was observed that the $d_{(001)}$ peaks largely shift to the left with decreasing $\mathrm{pH}$, indicating low $\mathrm{pH}$-facilitated intercalation, and its effect reached the best at $\mathrm{pH} 3$, where the interlayer distance between MMT platelets increased from 1.31 to $3.52 \mathrm{~nm}$. Thermogravimetric analysis showed that the modified sodium MMT exhibited excellent thermal stability; the onset and the maximum decomposition temperature were 343 and $406^{\circ} \mathrm{C}$ respectively.

\section{Introduction}

In recent years, polymer-layered silicate (PLS) nanocomposites have attracted wide attention. ${ }^{1}$ The layered silicate materials, used as nanoscale reinforcing agents in a polymer matrix, will cause significant improvements in mechanical, thermal, barrier and flame-retardant properties that have not yet been observed in any component. ${ }^{2}$ Therefore, it is desirable to make appropriate modification of the inherently hydrophilic montmorillonite (MMT) in the preparation of PLS nanocomposites.

The most commonly used organic modifiers are alkyl quaternary ammonium, ${ }^{3,4}$ amino acids ${ }^{5}$ and phosphonium cationic surfactants. The modification mechanism can be explained as follows: (a) These organic cations undergo ion-exchange reactions on exchangeable cations of MMT layers, and the organic groups will cover the surface of MMT or insert into the MMT layers, making the surface properties of MMT change from hydrophilic to lipophilic and the interlayer distance increase. (b) The interlayer force is weakened, and this facilitates intercalation. Although these modifiers have many advantages such as lower cost, commercial availability and the ability to render clays miscible with most polymer matrices, they do show poor thermal stability with decomposition temperature from 233 to $310^{\circ} \mathrm{C}^{6-8}$ VanderHart et al. ${ }^{9}$ found that decomposition will occur and alter the interface between the filler and the matrix polymer if the processing temperature is higher than the thermal stability of the organoclay. Accordingly, the thermal stability of the organic surfactant plays an important role in the preparation and further processing of PLS nanocomposites. Xie et al. ${ }^{6,7}$ reported that the onset decomposition temperature of MMT, modified with long carbon-chain alkyl quaternary ammonium ions, is approximately $180^{\circ} \mathrm{C}$. However, the typical melt temperature used in polymer processing is always beyond $200^{\circ} \mathrm{C}^{10,11}$ To overcome this problem, more stable cations, such as pyridinium and imidazolium salts, have been used to modify layered silicates due to their inherently higher thermal stability. $8,12,13$

Ngo et al. ${ }^{14}$ and Begg et al. ${ }^{15}$ found that imidazole exhibits resistance to ring fission during thermal rearrangements of 1-alkyl- and 1-aryl-imidazoles at temperatures above $600^{\circ} \mathrm{C}$, which indicates that the imidazolium cation is more thermally stable than the alkyl ammonium cation. Experimental results ${ }^{8,16-19}$ 
concerning imidazolium-intercalated MMT showed an increase in thermal stability as compared to ammonium-modified MMT. He et $a .^{20}$ reported that the initial decomposition temperature (temperature at $5 \%$ weight loss $T_{0.05}$ ) of 1-hexadecane3-methylimidazolium bromine, 1-hydroxyethyl-3-hexadecane imidazolium bromine and 1,3-dihexadecane imidazolium iodine with saturated alkyl groups was higher than $250^{\circ} \mathrm{C}$. The $T_{0.05}$ values of organic montmorillonite (oMMT) were higher than $330^{\circ} \mathrm{C}$. In addition, it was found that dihexadecane imidazolium, with two long tails, has the ability to enlarge the interlayer distance to a higher degree as compared to other imidazolium surfactants with only one long tail. Shailesh $e t a .^{21}$ found that the onset decomposition temperature $\left(T_{\text {onset }}\right)$ of alkyl-imidazolium salt 1-decyl-2-methyl-3-(11-carboxyundecyl) imidazolium bromide reached $271^{\circ} \mathrm{C}$ and the $T_{\text {onset }}$ of alkyl-imidazolium-treated layered silicates reached $376^{\circ} \mathrm{C}$. The advantageous features of the imidazolium compounds include the imidazole ring offering the compound high thermal stability as compared to that of alkylammonium compounds, and various alkyl substitute groups resulting in improved hydrophobicity of clay and enlarged gallery of silicate layers. Therefore, the authors of the present paper considered the imidazolium salts as a surfactant for modifying layered silicates owing to their inherently greater thermal stability.

The objective of this work was to synthesize thermally stable alkyl imidazolium salts as MMT modification surfactant. Nuclear magnetic resonance (NMR) and Fourier transform infrared spectroscopy (FTIR) were used to characterize the synthesized surfactant. oMMT, modified with this surfactant, was prepared by ion exchange. The intercalation effect and the thermal stability of oMMT were specifically investigated. Moreover, the modification medium as function of $\mathrm{pH}$ was also studied.

\section{Experimental}

\subsection{Materials}

The raw materials of 1-bromohexadecane $\left(\mathrm{C}_{16} \mathrm{H}_{33} \mathrm{Br}\right)$ supplied by Shanghai Aladdin Co. Ltd, 1,2-dimethyl imidazole bought from Shanghai Lingfeng Chemical Reagent Co. Ltd and acetonitrile purchased from Sinopharm Chemical Reagent Co. Ltd were used to form the organic modifier. The solvent ethyl acetate, from Sinopharm Chemical Reagent Co. Ltd, was used to precipitate and wash the synthetic product. Sodium (Na) MMT, with a cation-exchange capacity of $80 \mathrm{meq} / 100 \mathrm{~g}$, was provided by Zhejiang Fenghong New Material Co. Ltd, and silver nitrate $\left(\mathrm{AgNO}_{3}\right)$ was supplied by Sinopharm Chemical Reagent Co. Ltd. All reagents used were of analytical reagent grade and used without any purification.

\subsection{Preparation of 1-hexadecyl-2,3- dimethylimidazolium bromide}

Given the volatility of bromoethane, the molar ratio of 1,2dimethyl imidazole and alkyl bromide was $1: 1 \cdot 1$, while some acetonitrile was added. The mixture was firstly placed in a thickwalled single-necked round-bottom flask equipped with a reflux condenser tube, a thermometer and a magnetic stirrer. Then the mixture was stirred in a water $\left(\mathrm{H}_{2} \mathrm{O}\right)$ bath for $12 \mathrm{~h}$ at $90^{\circ} \mathrm{C}$ in high-purity nitrogen flowing atmosphere. After completion of reaction, the product was cooled to room temperature. In order to remove all unreacted 1,2-dimethyl imidazole, the product was subsequently precipitated, washed and filtrated for several times with ethyl acetate. The wet product was dried in vacuum at $80^{\circ} \mathrm{C}$ for $24 \mathrm{~h}$, and then the remaining ethyl acetate and pale yellow powdery solid were obtained. Afterwards, the pale yellow powdery solid was dissolved with a small amount of acetonitrile and was precipitated with ethyl acetate to obtain a white solid. The white solid was then washed with ethyl acetate and filtered several times and dried in vacuum at $80^{\circ} \mathrm{C}$ for $24 \mathrm{~h}$. Finally, the white powder, 1-hexadecyl-2,3-dimethylimidazolium bromide, was obtained.

\subsection{Organic montmorillonite}

oMMT was prepared based on the cation-exchange procedure. ${ }^{22}$ Distilled water $(125 \mathrm{ml})$, used as a dispersant medium, was added to a beaker and heated to $80^{\circ} \mathrm{C}$ in an oil bath. At the same time, the $\mathrm{pH}$ was regulated to set values of $1,3,5,7,9,11$ and 13 and sodium MMT was then added. The mixture was stirred at a high speed to make sodium MMT disperse totally in the distilled water. A certain amount of 1-hexadecyl-2,3dimethylimidazolium bromide was then completely dissolved in hot distilled water and added into the emulsion. Subsequently, the mixture was stirred for $5 \mathrm{~h}$ at $80^{\circ} \mathrm{C}$ at 800 revolutions per minute and then set aside for $12 \mathrm{~h}$. Removing the foam and the liquid, a milky white flocculent precipitate was obtained. The oMMT was collected by filtration and washed several times with hot distilled water to remove residual salts. Washing was continued until no halide anion was detected by the silver nitrate test. Finally, the oMMT was dried at $80^{\circ} \mathrm{C}$ for $24 \mathrm{~h}$ in vacuum to constant weight and then milled to a fine powder, which was passed through a 200-mesh sieve.

\subsection{Characterization}

\subsubsection{Structure}

The structure of 1-hexadecyl-2,3-dimethylimidazolium bromide was characterized by FTIR and NMR. FTIR scans were performed in transmission mode by using potassium bromide $(\mathrm{KBr})$ pellets on a Thermo Nicolet Nexus 670 spectrometer while during NMR measurements, deuterated chloroform was used as a solvent and tetramethylsilane as the reference.

\subsubsection{X-ray diffraction}

The samples were analyzed by a Bruker D8 Advance X-ray diffraction (XRD) instrument with an incident X-ray wavelength of $1.541 \AA$ and operating at a voltage of $40 \mathrm{kV}$, a current of $40 \mathrm{~mA}$ and a scan rate of $2^{\circ} / \mathrm{min}$ from 1 to $4^{\circ}$.

\subsubsection{Transmission electron microscopy}

The interlayer distance of the modified oMMT was characterized by transmission electron microscopy (TEM). TEM images were 
obtained using a JEM-2100 STEM/EDS transmission electron microscope. The solvent of oMMT was an ethanol solution.

\subsubsection{Thermogravimetric analysis}

Thermogravimetric analysis (TGA) was carried out on neat oMMT to examine its thermal stability. The experiments were conducted using an STA449c/3/G TGA instrument supplied by Netzsch Company under nitrogen atmosphere. Samples (10-20 mg) were prepared and heated at a constant rate of $10^{\circ} \mathrm{C} / \mathrm{min}$ from 25 to $700^{\circ} \mathrm{C}$.

\section{Results and discussion}

\subsection{Characterization of 1-hexadecyl-2,3- dimethylimidazolium bromide}

The FTIR spectra of 1-hexadecyl-2,3-dimethylimidazolium bromide is shown in Figure 1. A doublet at 3478 and $3300 \mathrm{~cm}^{-1}$ is assigned to the $\mathrm{N}-\mathrm{H}$ stretching vibration in the imidazolium ring, and the band at about $1530 \mathrm{~cm}^{-1}$ is assigned to the $\mathrm{N}-\mathrm{H}$ deformation mode in the imidazolium ring. In addition, a band at $3062 \mathrm{~cm}^{-1}$ is caused by the $\mathrm{C}-\mathrm{H}$ stretching vibration of the imidazole ring, whereas the band at $2915 \mathrm{~cm}^{-1}$ is caused by the saturated $\mathrm{C}-\mathrm{H}$ stretching vibration. The band at $1571 \mathrm{~cm}^{-1}$ is assigned to the $\mathrm{C}=\mathrm{C}$ vibration in the imidazolium ring, the band at $1180 \mathrm{~cm}^{-1}$ is assigned to the $\mathrm{C}-\mathrm{Br}$ vibration and the bands at 622 and $717 \mathrm{~cm}^{-1}$ are assigned to $-\left(\mathrm{CH}_{2}\right)_{15}$ bending vibration.

The NMR spectra of 1-hexadecyl-2,3-dimethylimidazolium bromide is shown in Figure 2. It is found that $\delta$ between 0.86 and 2.71 represent various hydrogens of 16 alkyl, $\delta=4.13$ represents $-\mathrm{NCH}_{3}, \delta=4.31$ represents $-\mathrm{CCH}_{3}$ and $\delta=7 \cdot 37, \delta=7 \cdot 55$ and $\delta=10 \cdot 4$ represent $\mathrm{H}^{5}, \mathrm{H}^{4}$ and $\mathrm{H}^{2}$ of the imidazole ring respectively.

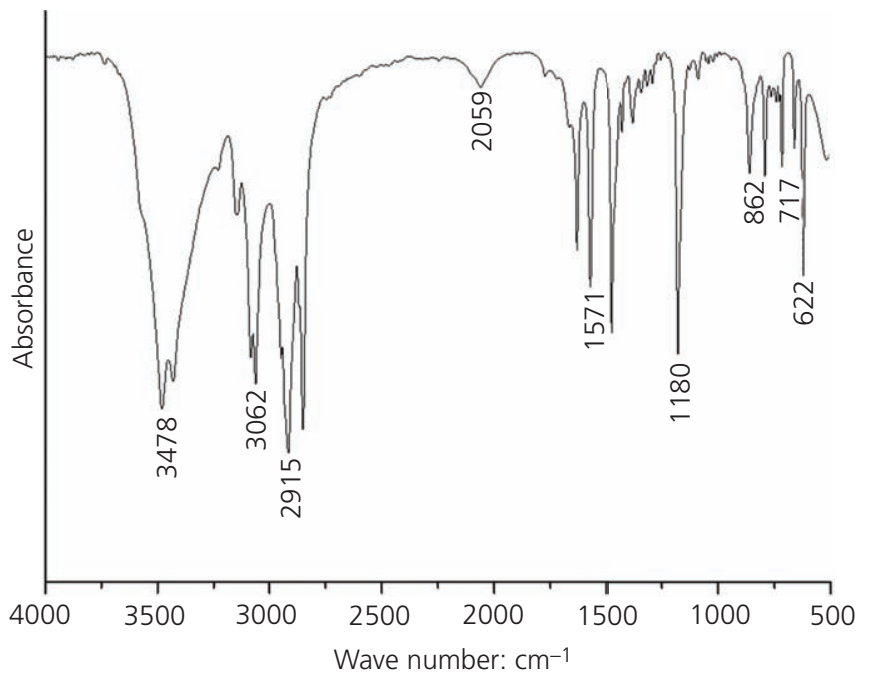

Figure 1. FTIR spectra of 1-hexadecyl-2,3-dimethylimidazolium bromide

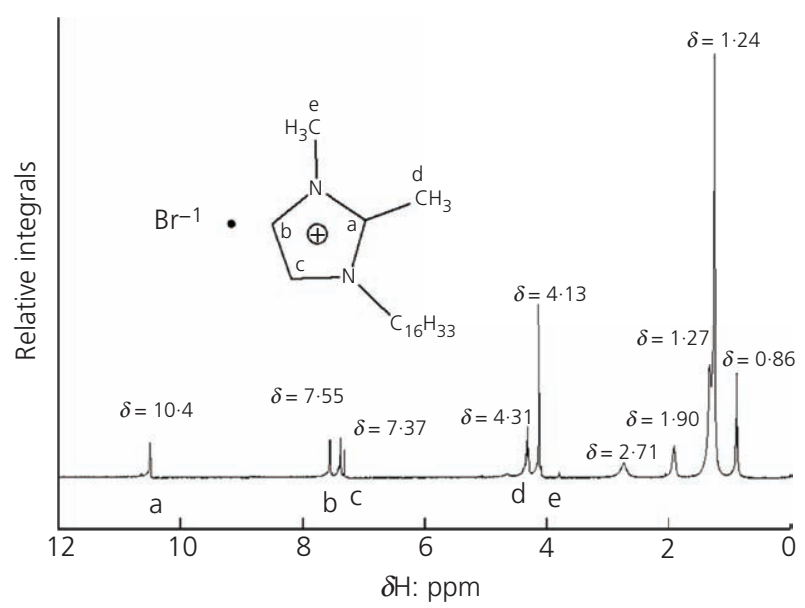

Figure 2. Proton $\left({ }^{1} \mathrm{H}\right)$ NMR spectra of 1-hexadecyl-2,3dimethylimidazolium bromide

\subsection{Characterization of oMIMT}

\subsubsection{Fourier transform infrared spectroscopy}

The FTIR spectra of MMT and imidazolium oMMT are shown in Figure 3. The bands at 3620 and $3420 \mathrm{~cm}^{-1}$ are assigned to the $-\mathrm{OH}$ stretching vibration. The $\mathrm{Si}-\mathrm{O}$ stretching band is observed at $1045 \mathrm{~cm}^{-1}$, and a band at $516 \mathrm{~cm}^{-1}$ is assigned to the $\mathrm{Si}-\mathrm{O}$ bending vibration. A band at $466 \mathrm{~cm}^{-1}$ is assigned to the $\mathrm{Al}-\mathrm{O}$ bending vibration.

From the FTIR spectra of oMMT, all the bands belonging to typical MMT are reserved after modification, and this indicates the fact that MMT has been modified by the surfactant. A pair of bands at 2927 and $2850 \mathrm{~cm}^{-1}$ is assigned to the asymmetric and symmetric $\mathrm{CH}_{2}$ stretching vibrations. A band at $1631 \mathrm{~cm}^{-1}$ is assigned to the $\mathrm{C}-\mathrm{N}$ stretching vibration. These bands are absent in MMT, and this suggests that the cationic surfactants have been intercalated into the interlayer of sodium MMT to form oMMT

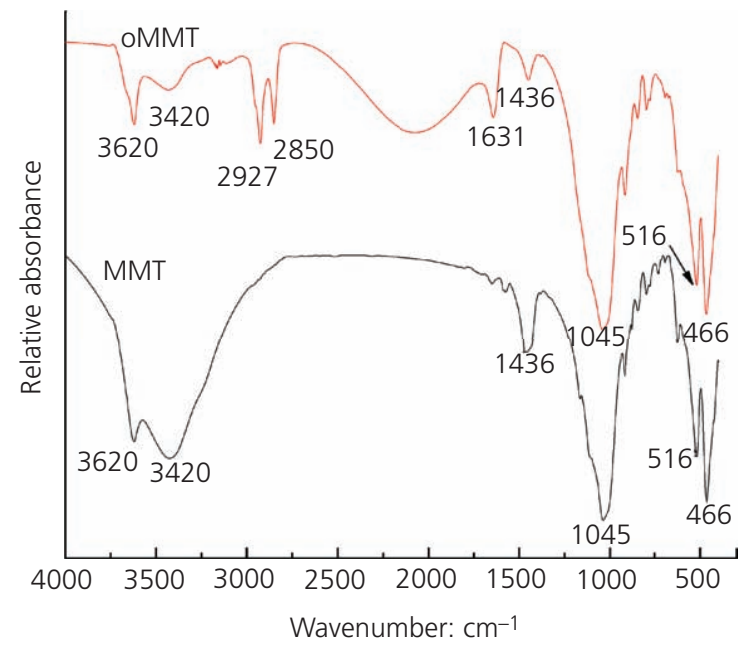

Figure 3. FTIR spectra of imidazolium-based sodium MMT 


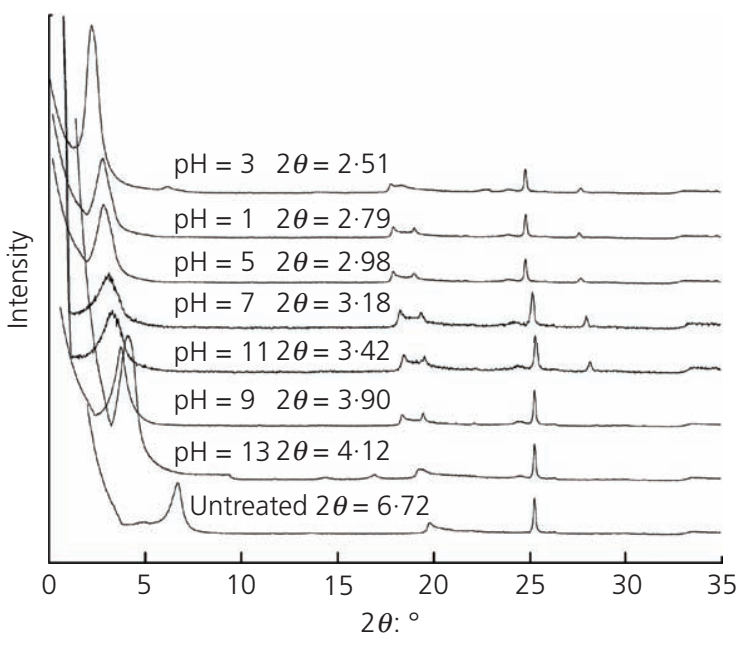

Figure 4. XRD spectra of oMMT treated at various $\mathrm{pH}$ values

\subsubsection{The intercalation effect on different $\mathrm{pH}$ values}

For PLS nanocomposites, the interlayer distance of MMT is an important parameter for evaluating the treatment efficiency of oMMT and the type of the PLS nanocomposites. Therefore, it is imperative to obtain the interlayer distance during the process of preparation. The interlayer distances between the layers of MMT are calculated by using the Bragg equation with the diffraction angle $\theta$ of (001).

The Bragg equation is given as

1. $2 d \sin \theta=n \lambda$

where $d$ is the interlayer distance (nm), $\theta$ is the Bragg angle $\left(^{\circ}\right), n$ is the reflection coefficient and $\lambda$ is the wavelength (nm).

The XRD spectra of oMMT treated at various $\mathrm{pH}$ values are shown in Figure 4. It is observed that the change in $\mathrm{pH}$ has a significant influence on oMMT and the acidic medium favors intercalation. MMT is made of flaky clay particles with three kinds of charges: ${ }^{23}$ a permanent negative charge, a variable negative charge and a positive charge. The type and the quantity of charges vary with the type of MMT. The permanent negative charge, constituting more than $95 \%$, is determined by the MMT itself, and its quantity remains stable under various $\mathrm{pH}$ values. In the alkaline medium, a hydrogen ion $\left(\mathrm{H}^{+}\right)$which is dissociated from MMT combines with a hydroxyl ion $\left(\mathrm{OH}^{-}\right)$in the medium to generate water. Thus, the surface of MMT forms negative charges - that is, the variable negative charge.

In the acidic medium, a hydroxyl ion which is dissociated from MMT combines with a hydrogen ion in the medium to generate water, so that the surface of MMT is positively charged. In the alkaline medium, the intercalation effect decreases with increasing $\mathrm{pH}$ values. However, in an acidic medium, the intercalation effect increases and then decreases with decreasing $\mathrm{pH}$ values. The $d_{(001)}$ peaks largely shift to the left with decreasing $\mathrm{pH}$ in Figure 4. The intercalation effect is the best at $\mathrm{pH} \mathrm{3,} \mathrm{where} \mathrm{the}$ interlayer distance reaches $3.52 \mathrm{~nm}$ (see Table 1). The reason is as follows: in the alkaline medium, imidazole salt cations are affected by the end face negative charge and adsorbed on both ends of the lamella, which makes it difficult for organic cations to be inserted into layers of MMT. However, in the acidic medium, the end face positive charge has a repulsive force against imidazole salt cations. Thus, the organic cation can be inserted into the layers of MMT easily and replace hydrated cations to achieve a better intercalation effect. Therefore, the acidic medium favors intercalation of MMT.

In addition, Figure 5 (TEM images) offers the most explicit visualization of the intercalation effect. Figures 5(a) and 5(b) are for MMT and oMMT treated at $\mathrm{pH} 3$ respectively. It can be seen that the interlayer distance increases from 1.15 to $3.45 \mathrm{~nm}$, and the results are consistent with those shown by XRD.

\subsubsection{Thermal stability of oMMT}

The degradation of oMMT(DMHDIM-MMT) modified by 1-hexadecyl-2,3-dimethylimidazolium bromide and oMMT (DMDODA-MMT) modified by dimethyl dioctadecyl ammonium bromide were monitored by thermogravimetric analysis from 25 to $700^{\circ} \mathrm{C}$; the results are shown in Figure 6 . The signals from the TGA have been scaled to represent the remaining weight percentage of the modifier originally on the oMMT, since the inorganic constituents in MMT remain stable and its weight will not change during the tests at the used temperature. As expected, the differences in the thermal stability of these two oMMTs are remarkable. Both modifiers have one long alkyl tail; however,

\begin{tabular}{|c|c|c|c|c|c|c|c|c|}
\hline & \multicolumn{7}{|c|}{ pH } & \multirow{2}{*}{ Untreated } \\
\hline & 1 & 3 & 5 & 7 & 9 & 11 & 13 & \\
\hline $2 \theta$ angle: ${ }^{\circ}$ & $2 \cdot 79$ & $2 \cdot 51$ & 2.98 & $3 \cdot 18$ & $3 \cdot 42$ & 3.90 & $4 \cdot 12$ & $6 \cdot 72$ \\
\hline$d_{(001)}: n m$ & $3 \cdot 16$ & 3.52 & 2.96 & $2 \cdot 77$ & $2 \cdot 58$ & $2 \cdot 26$ & $2 \cdot 14$ & $1 \cdot 31$ \\
\hline
\end{tabular}

Table 1. Results of interlamellar spaces and angles of oMMT with

different $\mathrm{pH}$ values 
Emerging Materials Research Volume 5 Issue EMR2
Thermal analysis of montmorillonite

modified by imidazolium

Zhang, Mei, Chen, Chen and Zu

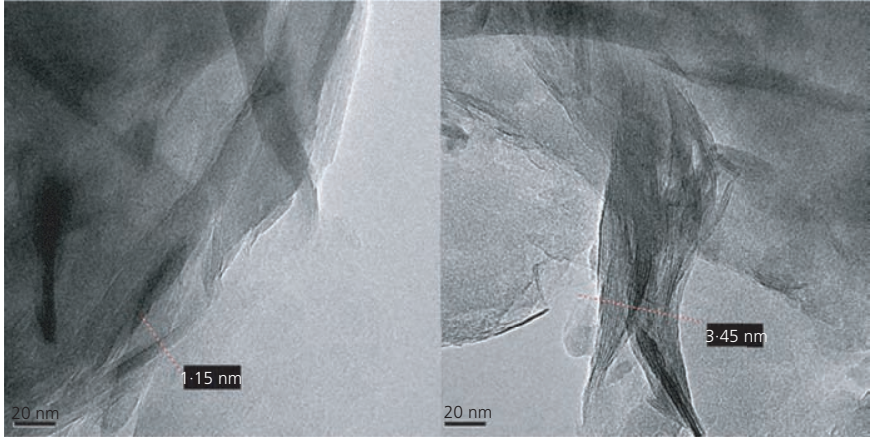

(a)

(b)

Figure 5. TEM micrographs of (a) MMT and (b) OMMT

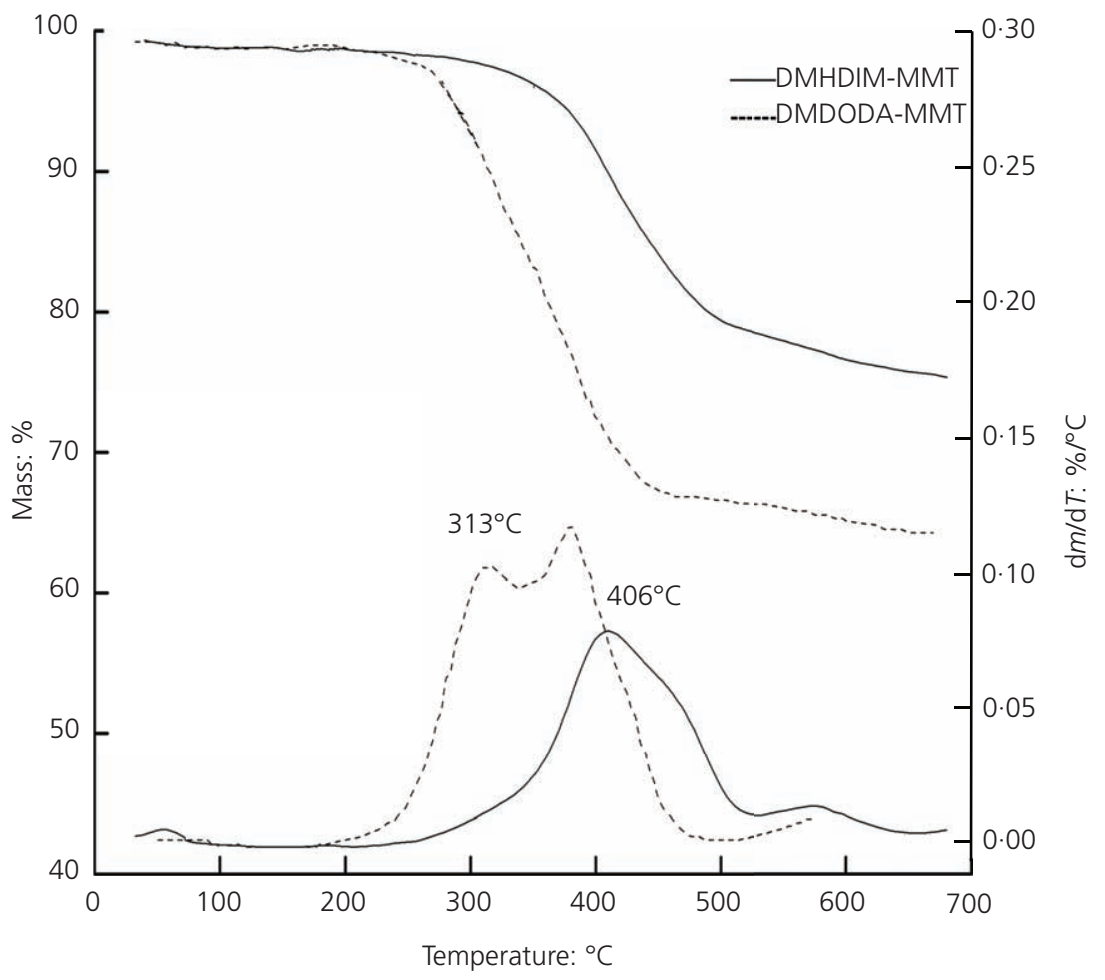

Figure 6. Comparison of TGA results for DMHDIM-MMT and DMDODA-MMT

\begin{tabular}{|c|c|c|c|c|}
\hline Type of organomodifier & $T_{\text {onset }}:{ }^{\circ} \mathrm{C}$ & $T_{\text {max }}:{ }^{\circ} \mathrm{C}$ & Change in $d: \mathbf{n m}$ & Reference \\
\hline 1-Hexadecyl-2,3-dimethylimidazolium bromide & 343 & 406 & $2 \cdot 21$ & - \\
\hline Dimethyl dioctadecyl ammonium bromide & 280 & 308 & $1 \cdot 49$ & 8 \\
\hline Hexadecyl-tributyl phosphorus bromide & 320 & 378 & $2 \cdot 01$ & 8 \\
\hline
\end{tabular}

Table 2. Thermal stability parameters and change in $d$ of various oMMTs 
their cations are imidazolium and ammonium types respectively. Obviously, the MMT modified using the imidazolium base is much more thermally stable than that modified with the ammonium base.

Figure 6 shows that the imidazolium-modified MMT begins to decompose at $343^{\circ} \mathrm{C}$ and the peak decomposition temperature is $406^{\circ} \mathrm{C}$, whereas the ammonium-modified MMT begins to decompose at $280^{\circ} \mathrm{C}$ and the peak decomposition temperature is $313^{\circ} \mathrm{C}$. The comparison of thermal stability parameters among three kinds of oMMTs ${ }^{8}$ is given in Table 2. Generally, the alkyl quaternary ammonium salts will show thermal degradation at $200^{\circ} \mathrm{C}$ either by a Hofmann elimination to give a product that is different from the amine or by an $\mathrm{S}_{\mathrm{N}} 2$ nucleophilic substitution reaction to produce the amine. ${ }^{6}$ Higher thermal stability of imidazolium-modified MMT is attributed to the imidazole ring in the imidazole salt.

\section{Conclusions}

An imidazolium surfactant - that is, 1-hexadecyl-2,3dimethylimidazolium bromide - was prepared and used to improve the properties of sodium MMT. The structure of 1-hexadecyl-2,3-dimethylimidazolium bromide was confirmed by FTIR and NMR spectroscopy. The cation-exchange reaction, using imidazole salts, was performed on sodium MMT. oMMT was characterized by powder XRD and FTIR techniques to confirm exchange. The intercalation of the imidazolium surfactant in sodium MMT certainly increased the basal spacing of MMT and the low $\mathrm{pH}$ favored intercalation. The intercalation effect reached its best value at $\mathrm{pH} 3$. The interlayer distance increased from 1.31 to $3.52 \mathrm{~nm}$. The sodium MMT, based on the imidazolium surfactant, was demonstrated to have excellent thermal stability, which is significantly greater than that of the ammonium surfactant. In addition, the onset and the maximum decomposition temperature were 343 and $406^{\circ} \mathrm{C}$ respectively.

\section{Acknowledgements}

This work was supported by the National Natural Science Foundation of China (Grant Numbers 51173141/E030701).

\section{REFERENCES}

1. Pinnavaia TJ and Beall GW (2000) Polymer-Clay Nanocomposites. Wiley, New York, NY, USA.

2. Giannelis EP (1996) Polymer layered silicate nanocomposites. Advanced Materials 8(1): 29-35.

3. Weimer WM, Chen H, Giannelis EP et al. (1999) Direct synthesis dispersed nanocomposites by in situ living free radical polymerization using a silicate-anchored initiator. Journal of American Chemical Society 121(7): 1615-1616.

4. Fu X and Qutubuddin S (2001) Polymer-clay nanocomposites: exfoliation of organophilic montmorillonite nanolayers in polystyrene. Polymer 42(2): 807-813.

5. Okada A, Kawasumi M, Kurauchi T et al. (1987) Synthesis and characterization of a nylon 6-clay hybrid. Polymeric Preprints 28: 447-448.
6. Xie W, Gao Z, Pan W et al. (2001) Thermal degradation chemistry of alkyl quaternary ammonium montmorillonite. Chemistry of Materials 13(9): 2979-2990.

7. Xie W, Gao Z, Liu K et al. (2001) Thermal characterization of organically modified montmorillonite. Thermochimica Acta 367: $339-350$.

8. Gilman JW, Awad WH, Davis RD et al. (2002) Polymer/ layered silicate nanocomposites from thermally stable trialkyl imidazolium-treated montmorillonite. Chemistry of Materials 14(9): 3776-3785.

9. VanderHart DL, Asano A and Gilman JW (2001) Solid-state NMR investigation of paramagnetic nylon- 6 clay nanocomposites. Chemistry of Materials 13(10): 3796-3809.

10. Fornes TD, Yoon PJ and Paul DR (2003) Polymer matrix degradation and color formation in melt processed nylon 6/ clay nanocomposites. Polymer 44(24): 7545-7556.

11. Hughes K and Bohan J (2002) In Proceedings of the Fire Retardant Chemical Association Meeting, Lancaster, PA, USA. Fire Retardant Chemicals Association, pp. 85-89.

12. Takekoshi T, Khouri FF, Campbell JR, Jordan TC and Dai KH (1996) Layered Minerals and Compositions Comprising the Same. US Patent 5530052, Jun.

13. Liang ZM, Yin J and Xu HJ (2003) Polyimide/montmorillonite nanocomposites based on thermally stable, rigid-rod aromatic amine modifiers. Polymer 44(5): 1391-1399.

14. Ngo HL, LeCompte $K$, Hargens $L$ and McEwen AB (2000) Thermal properties of imidazolium ionic liquids.

Thermochimica Acta 357: 97-102.

15. Begg CG, Grimmett MR and Wethey PD (1973) The thermally induced rearrangement of 1-substituted imidazoles. Australian Journal of Chemistry 26(11): 2435-2446.

16. Awad WH, Gilman JW, Nyden M et al. (2004) Thermal degradation studies of alkyl-imidazolium salts and their application in nanocomposites. Thermochimica Acta 409(1): $3-11$.

17. Mittal V (2007) Gas permeation and mechanical properties of polypropylene nanocomposites with thermally-stable imidazolium modified clay. European Polymer Journal 43: 3727-3736.

18. Davis CH, Mathias LJ, Gilman JW et al. (2002) Effects of meltprocessing conditions on the quality of poly(ethylene terephthalate) montmorillonite clay nanocomposites. Journal of Polymer Science, Part B: Polymer Physics 40(23): 2661-2666.

19. Modesti M, Besco S, Lorenzetti A et al. (2007) ABS/clay nanocomposites obtained by a solution technique: influence of clay organic modifiers. Polymer Degradation and Stability 92(12): 2206-2213.

20. He A, Wang L, Yao W et al. (2010) Structural design of imidazolium and its application in $\mathrm{PP} /$ montmorillonite nanocomposites. Polymer Degradation and Stability 95(4): 651-655.

21. Shailesh KG, Smita G and Lon JM (2012) Thermally stable organically modified layered silicates based on alkyl imidazolium salts. Journal of Colloid and Interface Science 368(1): 366-371. 
22. Balazs AC, Singh C and Zhulina E (1998) Modeling the interactions between polymers and clay. Surfaces through Self-Consistent Field Theory Macromolecules 31: 8370-8381.
23. Zhu J, He H, Guo J et al. (2003) Comparative study on the aggregation of different long-chain alkyl ammonia in montmorillonite. Acta Mineralagica Sinica 23: 193-198.

\section{HOW CAN YOU CONTRIBUTE?}

To discuss this paper, please submit up to 500 words to the journal office at journal@ice.org.uk. Your contribution will be forwarded to the author(s) for a reply and, if considered appropriate by the editor-in-chief, it will be published as a discussion in a future issue of the journal.

ICE Science journals rely entirely on contributions from the field of materials science and engineering. Information about how to submit your paper online is available at www.icevirtuallibrary.com/page/authors, where you will also find detailed author guidelines. 